\title{
The Design of Mold Clamping Mechanisms with Two Dead-Position Configurations
}

\author{
Tzu-Hsia Chen ${ }^{1, *}$, Long-Chang Hsieh ${ }^{2}$ \\ ${ }^{1}$ Department of Mechanical and Computer-Aided Engineering, Feng Chia University, Taiwan \\ ${ }^{2}$ Department of Power Mechanical Engineering, National Formosa University, Taiwan \\ Received 28 April 2019; received in revised form 08 June 2019; accepted 11 July 2019 \\ DOI: https://doi.org/10.46604/aiti.2020.4164
}

\begin{abstract}
Blow-molding machines are divided into two types: (1) rotary type blow-molding machine, and (2) linear type blow-molding machine. Good kinematic characteristics of mold clamping mechanism is the goal pursued by manufacturers of blow-molding machine. Traditionally, the mold clamping mechanism has only one dead-position configuration for the mold in the closed position. When the mold is in the open position, the mold clamping mechanism does not have dead-position configuration. This study focuses on the design of mold clamping mechanisms with two dead-position configurations when the mold is in open and closed positions. Firstly, with reference to the existing patents, based on the Yan's creative design methodology, four design concepts of mold clamping mechanisms are synthesized. Then, according to the studies on kinematics of mechanisms, three mold clamping mechanisms with two dead-position configurations are also synthesized. The results of this paper will enhance the research and development $(R \& D)$ capability of the mold clamping mechanisms to improve industrial competitiveness.
\end{abstract}

Keywords: blow-molding machine, conceptual design, creative design, dead-position configuration, mold clamping mechanism

\section{Introduction}

Due to the reason that the usage of Polyethylene terephthalate (PET) bottles is more and more widely, how to increase the production of PET bottles has become a major issue for the manufacturers. The requirements of the blow-molding machine will be stricter than ever. In order to improve the stability of the production of PET bottles, reducing the vibration and noise during the production process, extending the life of the machine, it is necessary to analyze the kinematics of mold clamping mechanism. Good kinematic characteristics of mold clamping mechanism is the goal pursued by manufacturers. Blow-molding machines are divided into two types: (1) rotary type blow-molding machine as shown in Fig. 1 (a), and (2) linear type blow-molding machine as shown in Fig. 1 (b). The motion of linear blow-molding machine is linear and reciprocated. The mold clamping mechanisms for linear type blow-molding machine are simple, stable, and reliable. Figs. 2 (a) and 2 (b) show the linear type blow-molding machine and its mold clamping mechanism. Traditionally, the mold clamping mechanism of linear type blow-molding machine has only one dead-position configuration for the mold in the closed position. When the mold is in the open position, the mold clamping mechanism does not have dead-position configuration. It results poor kinematic characteristics (larger or infinite acceleration), and causes blow-molding machine unstable. For the mold clamping mechanisms with two dead-position configurations in open and closed positions, it will have better kinematic characteristics (smaller max. acceleration), and causes blow-molding machine more stable.

* Corresponding author. E-mail address: summer34134@ gmail.com

Tel.: +886-4-2451-7250 ext.3501 


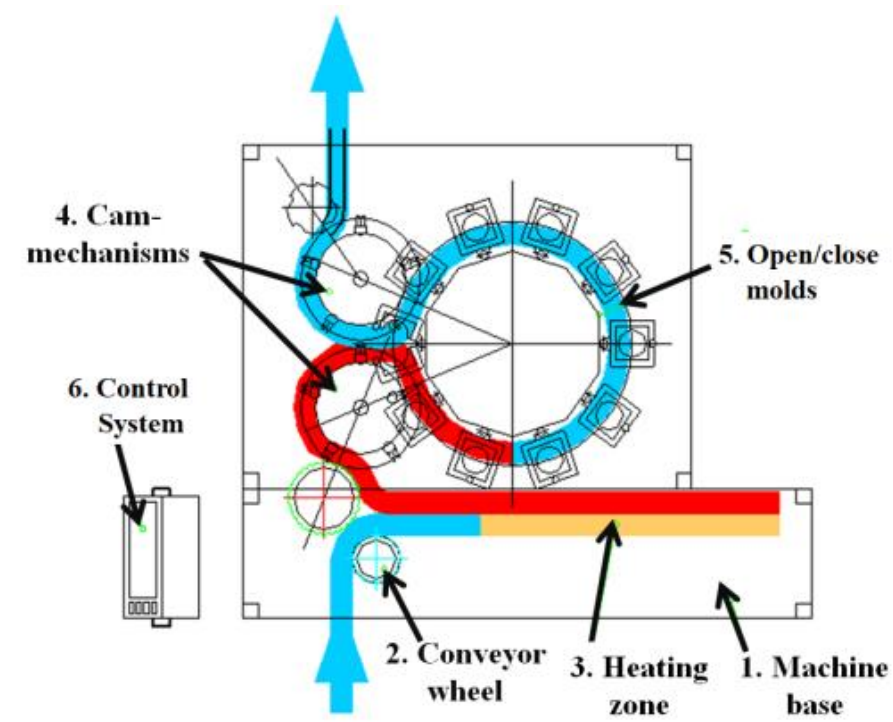

(a) Rotary type blow-molding machine

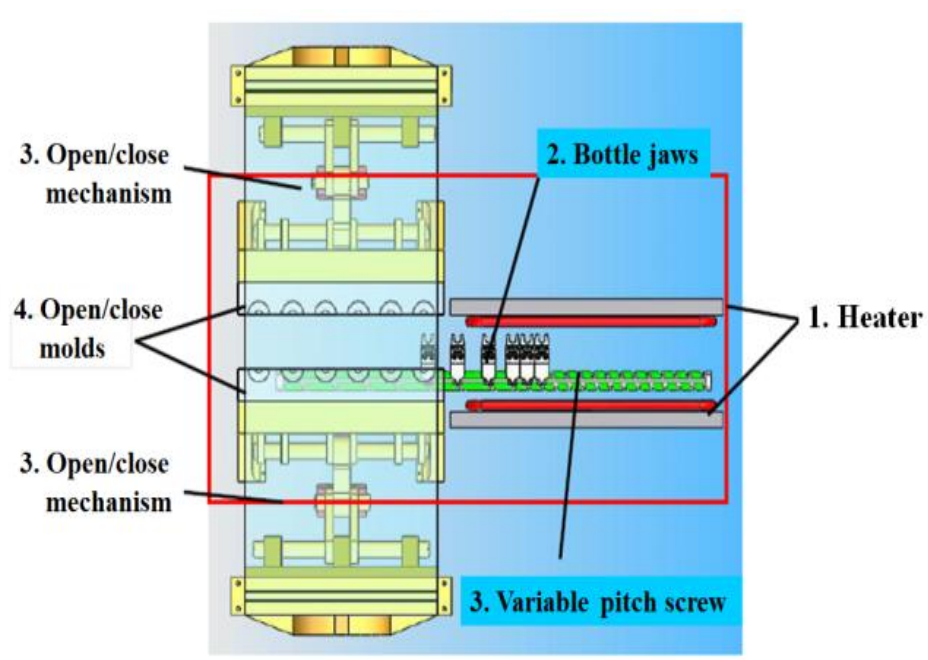

(b) Linear type blow-molding machine

Fig. 1 Types of blow-molding machines

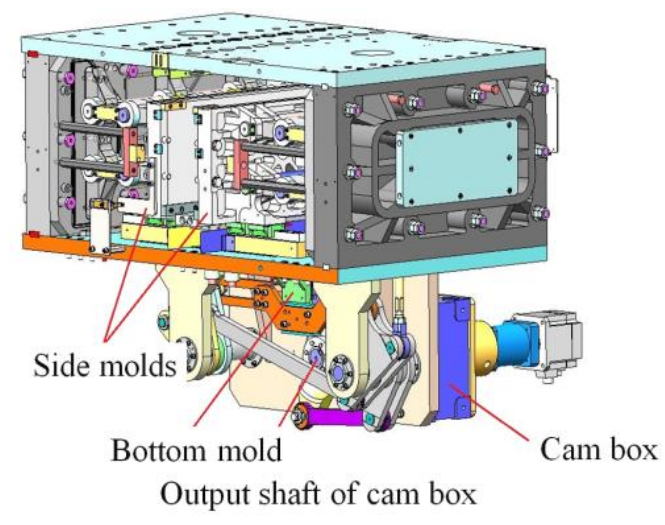

(a) Linear blow-molding machine

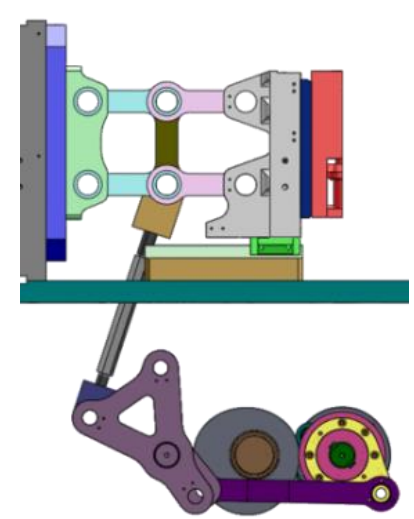

(b) Mold clamping mechanism

Fig. 2 Linear blow-molding machine

This study focuses on the design of mold clamping mechanisms with two dead-position configurations for linear blow-molding machine. Firstly, by referring to the existing patents [1-7] and the studies on the creative design [8-13], four feasible design concepts of mold clamping mechanisms are synthesized. Then, according to the studies on kinematics of mechanisms [14-20], three mold clamping mechanisms with two dead-position configurations are also synthesized. When the 
mold is in open and closed positions, the mold clamping mechanism will stay in the state of dead-position configuration (meanwhile, the mechanism is converted to desired structure). Hence, the mold clamping mechanisms with two dead-position configurations have the advantage of more safety. The results of this research can be used as reference when designing the real mold clamping mechanism and enhance R\&D capability of the mold clamping mechanisms to improve industrial competitiveness.

\section{Existing Design}

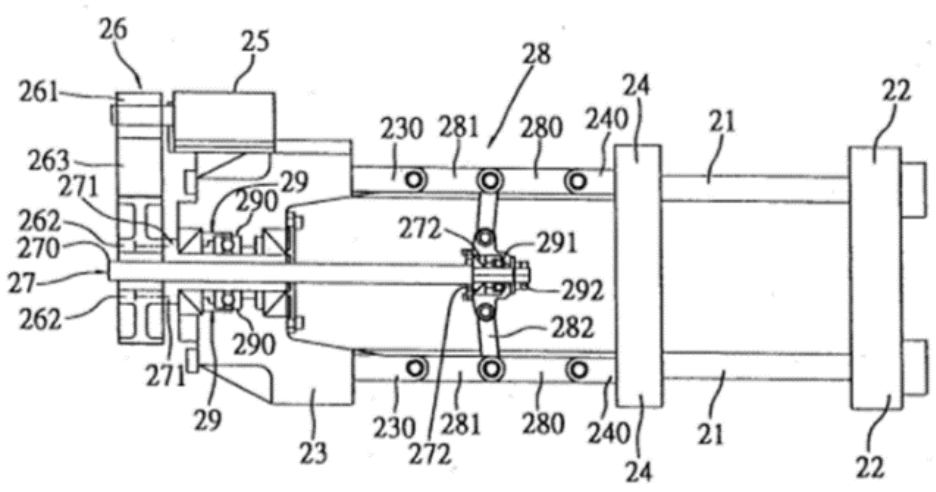

(a) Clamping mechanism

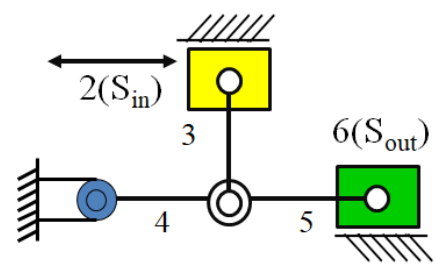

(b) Kinematic skeleton

Fig. 3 The mode clamping mechanism of servo-drive type injection molding machine [1]

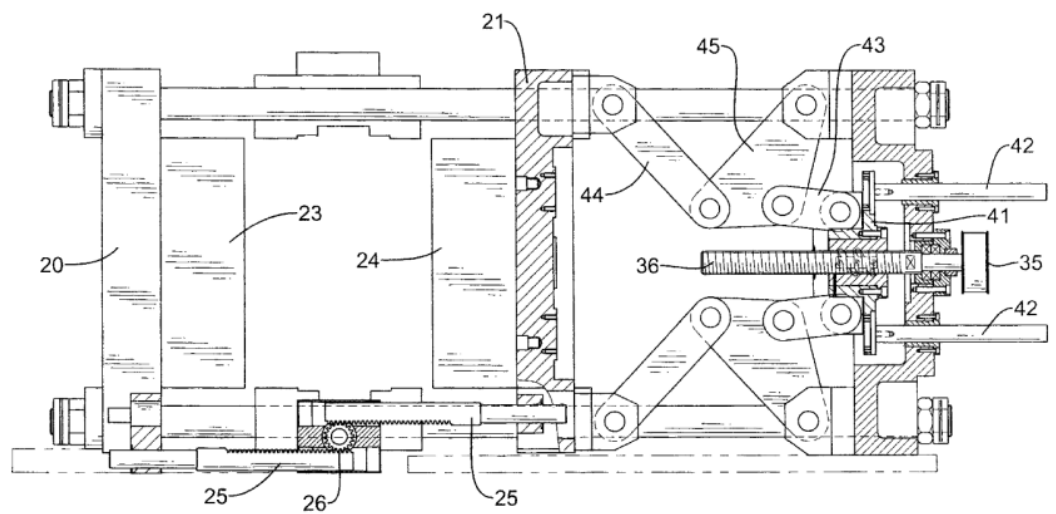

(a) Opening/closing mechanism

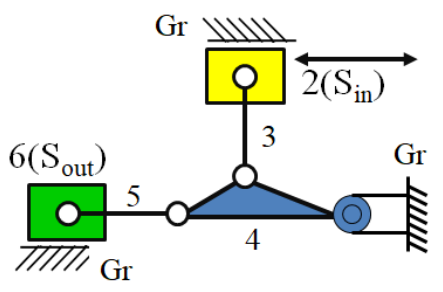

(b) Kinematic skeleton

Fig. 4 Driving device of controlling mode opening and closing for plastic molding machine [2] 
Lots of researches have been done about the topological characteristics for mold clamping mechanisms. Fig. 3 [1] and Fig. 4 [2] show planar mechanisms with 6 links and 7 joints.

For the mold clamping mechanism shown in Figs. 3 and 4, the mold clamping mechanism has a dead-position configuration when the mold is in the closed position. However, when the mold is in the open position, the mold clamping mechanism does not have dead-position configuration. The purpose of this paper is to design the mold clamping mechanism having dead-position configurations when the mold is in the open and closed positions.

The mobility of planar mechanism can be obtained by following equation.

$$
F=3(N-J-1)+\Sigma f i
$$

where $\mathrm{N}$ is number of links, $\mathrm{J}$ is number of joints, and fi is the degrees of freedom of joint $i$. The mechanisms, shown in Figs. 3 and 4, have 6 links, 7 joints ( 5 revolute pairs and 2 prismatic pair), according to equation of mobility, we get:

$$
F=3(N-J-1)+\Sigma f i=6(6-7-1)+7=+1
$$

The topological characteristics of mode clamping mechanisms for linear blow-molding machine are concluded as follows:

(1) It consists of 6 members and 7 joints.

(2) It has ground link (Gr, member 1), input slider $\left(S_{\text {in }}\right.$, member 2$)$, first connecting rod (member 3), rocker (member 4), second connecting rod (member 5), and output slider $\left(\mathrm{S}_{\text {out }}\right.$, member 6$)$.

(3) It has 7 joints including 5 revolute joints $\left(\mathrm{J}_{\mathrm{R}}\right)$ and 2 prismatic joints $\left(\mathrm{J}_{\mathrm{P}}\right)$.

(4) It is planar mechanism with 1 degree of freedom.

According to above reasoning, Fig. 5 shows the corresponding generalized chain of the patent [2] shown in Fig. 4.

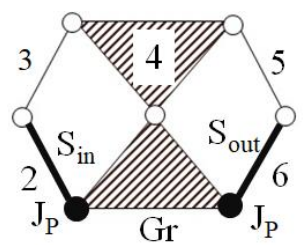

Fig. 5 Generalized chain of the mode clamping mechanism [2]

\section{Conceptual Design}

In 1998, Professor Yan proposed a creative design methodology [8], shown in Fig. 6, to create all feasible design concepts for mechanical devices. The steps are:

Step 1: Identify the topological characteristics based on the existing designs with required design specifications.

Step 2: Select any existing design and transform it into its corresponding generalized chain.

Step 3: Synthesize all generalized chains that have the same numbers of links and joints as the generalized chain obtained in Step 2.

Step 4: Assign types of links and joints to each generalized chain obtained in Step 3, to have the atlas of feasible specialized chains based on the algorithm of specialization to meet the design requirements and constraints.

Step 5: Particularize each feasible specialized chain obtained from Step 4 to its corresponding schematic format of mechanical device, to have the atlas of mechanical devices. 


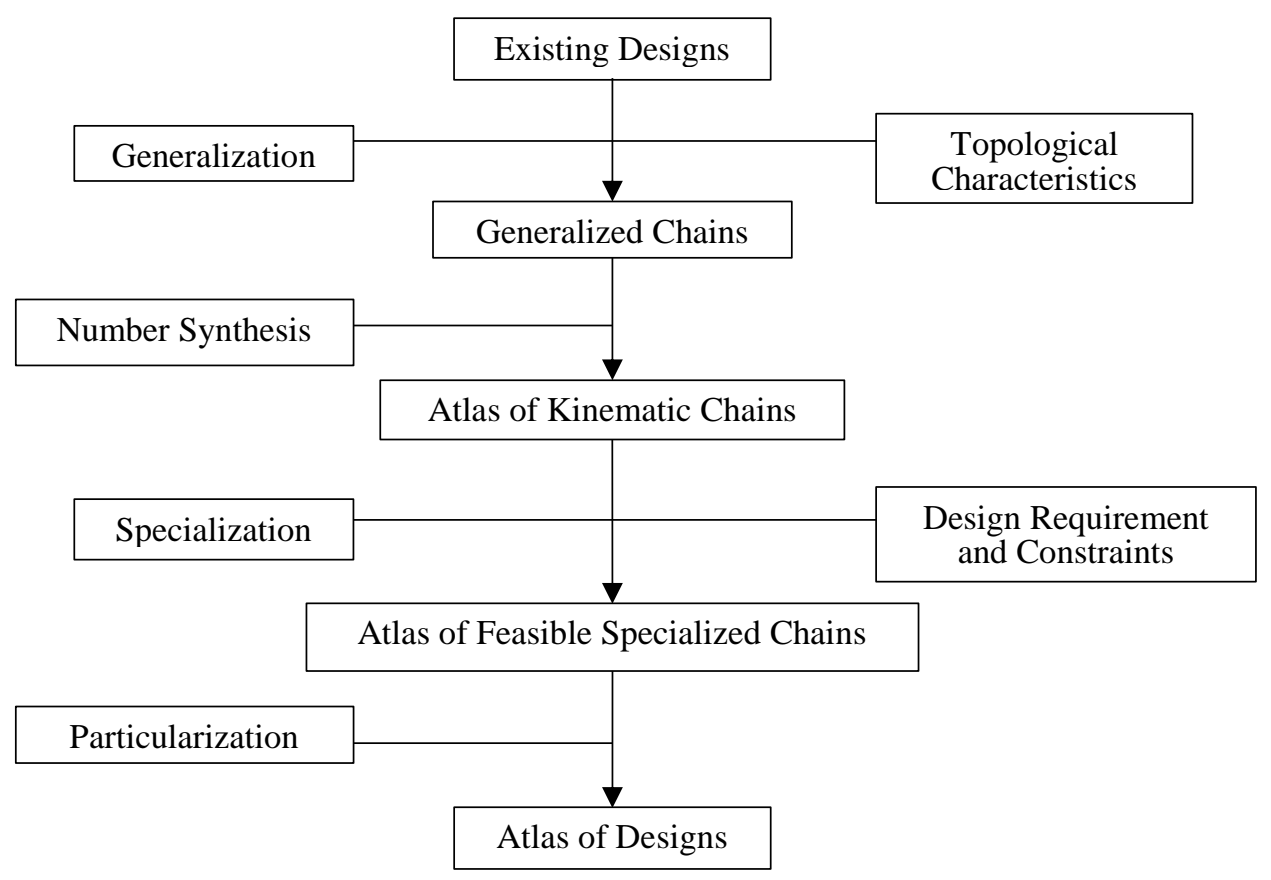

Fig. 6 Yan's creative design methodology [8]

\subsection{Atlas of kinematic chain}

According to the process of number synthesis, 2 kinematic chains with 6 links and 7 joints, shown in Fig. 7, are synthesized.

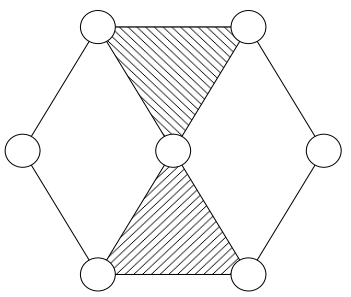

(a) 6-1

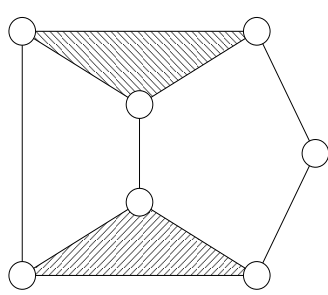

(b) 6-2

Fig. 7 Atlas of $(6,7)$ kinematic chains

\subsection{Design requirements and constrains}

Based on the concluded topological characteristics of existing designs, the design requirements and constrains of the mode clamping mechanisms are concluded as follow:

(1) There must be a ground link (Gr), input slider $\left(S_{\text {in }},\right)$, output slider $\left(S_{\text {out }}\right)$.

(2) The ground link as the frame must be ternary link.

(3) The ground link $(\mathrm{Gr})$ must be adjacent to input slider $\left(\mathrm{S}_{\mathrm{in}}\right)$ and output slider (opening/closing mold, $\left.\mathrm{S}_{\text {out }}\right)$ with prismatic pair.

(4) Input slider $\left(S_{\text {in }}\right)$ and output slider $\left(S_{\text {out }}\right)$ can't be in the same circuit with 1 degree of freedom.

(5) The mode clamping mechanism only includes prismatic pair $\left(\mathrm{J}_{\mathrm{P}}\right)$ and revolute pair $\left(\mathrm{J}_{\mathrm{R}}\right)$.

(6) The mode clamping mechanism must have 2 prismatic pairs.

\subsection{Specialization}

Subject to certain design requirements and constraints, the specializing steps for mode clamping mechanism are concluded as follow: 
(1) For each generalized (kinematic) chain, identify the ground link (Gr) for all possible cases.

(2) For each case obtained in step 1 , identify input slider $\left(S_{\text {in }}\right.$, denoted by thick black line and output slider $\left(\mathrm{S}_{\mathrm{out}}\right.$ denoted by thick black line

(3) For each case obtained in Step 3, identify the corresponding revolute pair (denoted by $\circ$ ) and prismatic pair (denoted by •).

Based on the design requirements and constraints, 3 feasible specialization chains, shown in Figs. 8(a), 8(b), and 8(c), are generated by the process of specialization.

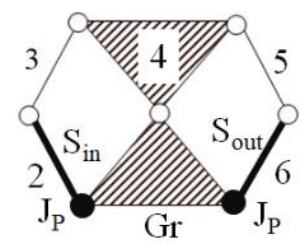

(a) 6-1-1

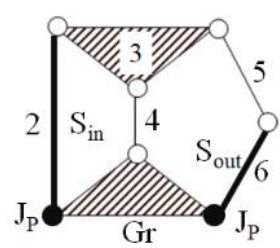

(b) 6-2-1

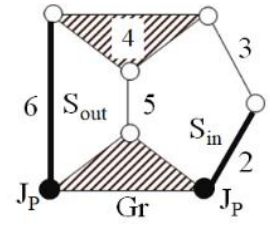

(c) $6-2-2$

Fig. 8 Atlas of $(6,7)$ feasible specialized chains

\subsection{Particularization}

For each feasible specialized chain, it can be particularized into its corresponding kinematic skeleton by the reverse process of generalization. For the feasible specialized chains shown in Figs. 8 (a) $\sim 8$ (c), their corresponding kinematic skeletons of mode clamping mechanisms are shown in Figs. 9 (a) 9 (c). According to the kinematic skeletons shown in Figs. 9 (a) $~ 9$ (c), one possible multiple-joint mechanism is synthesized and shown in Fig. 9 (d).

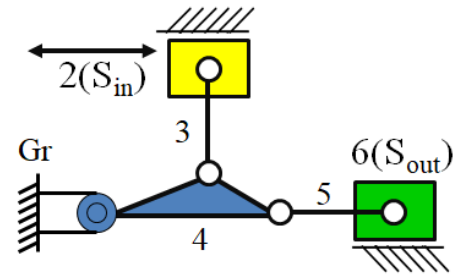

(a) Mechanisms (I)

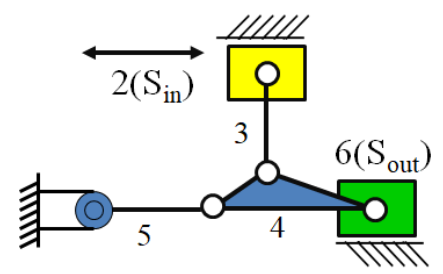

(c) Mechanisms (III)

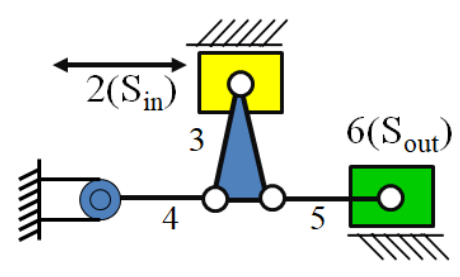

(b) Mechanisms (II)

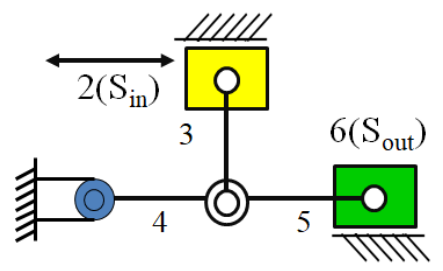

(d) Mechanisms (IV)

Fig. 9 Atlas of feasible mold clamping mechanisms of $(6,7)$ generalized chain

\section{Clamping Mechanisms with two Dead-Position Configurations}

\subsection{Case I}

Fig. 10 (a) and 10 (b) show the mode clamping mechanism (I) and its corresponding vector coordinate system of the mechanism shown in Fig. 9 (a). According to Fig. 10 (b), the mechanism has two independent vector loops (vector loops 1 and 2). Vector loops 1 and 2 can be expressed as follows:

$$
\vec{r}_{2}+\vec{r}_{3}-\vec{r}_{4 a}=0
$$




$$
\vec{r}_{4 b}+\vec{r}_{5}-\vec{r}_{6}=0
$$

According to Fig. 10 (b), $\theta_{4 a}=\theta_{4 b}+\alpha$ and $\theta_{4 c}=\theta_{4 a}-\pi+\beta$ are the given conditions. If we substitute them into the above equations, then the components $\mathrm{X}$ and $\mathrm{Y}$ can be expressed as:

$$
\begin{aligned}
& r_{2 x}+r_{3} \cos \theta_{3}-r_{4 a} \cos \theta_{4 a}=0 \\
& r_{2 y}+r_{3} \sin \theta_{3}-r_{4 a} \sin \theta_{4 a}=0 \\
& r_{4 b} \cos \theta_{4 b}+r_{5} \cos \theta_{5}-r_{6}=0 \\
& r_{4 b} \sin \theta_{4 b}+r_{5} \sin \theta_{5}=0
\end{aligned}
$$

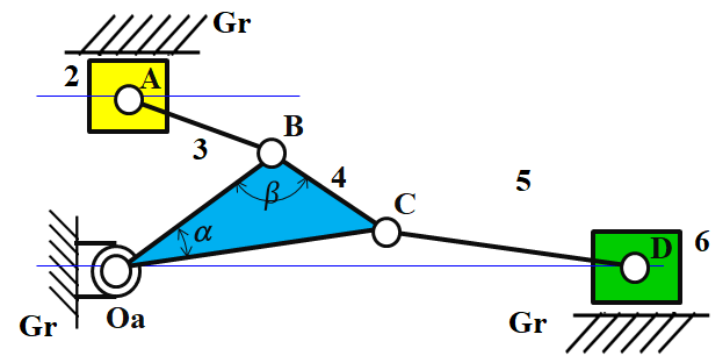

(a) Kinematic skeleton

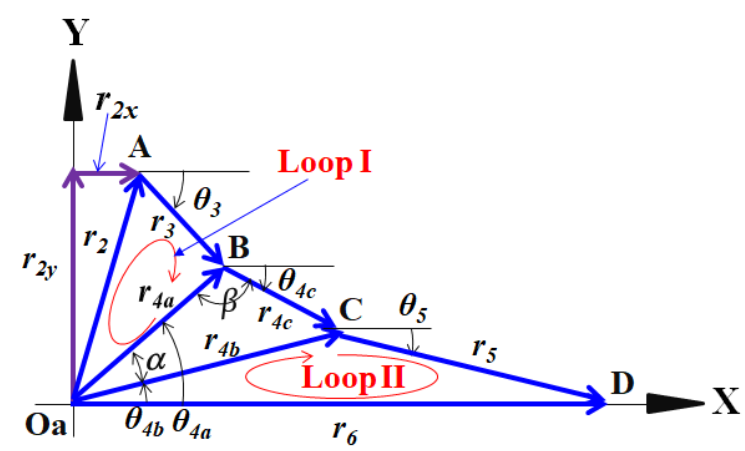

(b) Vector coordinate system

Fig. 10 Mold clamping mechanism (I)

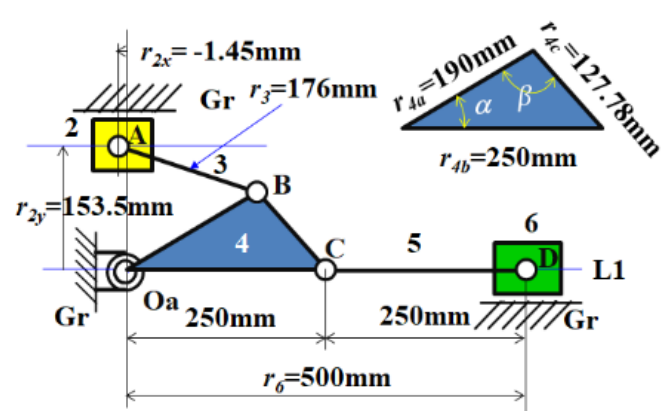

(a) Closed position (dead-position configuration, line L1)

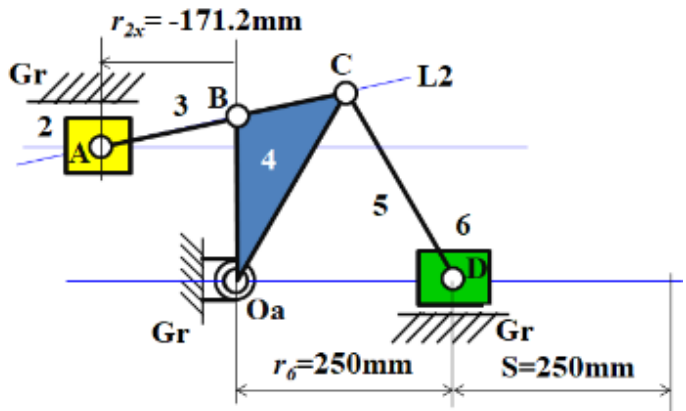

(b) Open position (dead-position configuration, line L2)

Fig. 11 Mold clamping mechanism (I) with two dead-position configurations

If $r_{2 y}=153.5 \mathrm{~mm}, r_{3}=176 \mathrm{~mm}, r_{4 a}=190 \mathrm{~mm}, r_{4 b}=r_{5}=250 \mathrm{~mm}, \alpha=30^{\circ}$, then $r_{4 c}=127.78 \mathrm{~mm}$ and $\beta=101.97^{\circ}$. According to Eqs. (5) (8), we get:

(1) If $\theta_{4 b}=0^{\circ}\left(\theta_{4 a}=30^{\circ}\right)$, then $\theta_{3}=-19.41^{\circ}, r_{2 x}=-1.45 \mathrm{~mm}, \theta_{4 c}=\theta_{4 a}-\pi+\beta=-48.03^{\circ}, \theta_{5}=0^{\circ}$, and $r_{6}=500 \mathrm{~mm}$. Fig. 11 (a) shows the corresponding dead-position configuration (line $\mathrm{L}_{1}$ ) of mode clamping mechanism at closed position.

(2) If $\theta_{4 b}=60^{\circ}\left(\theta_{4 a}=90^{\circ}\right)$, then $\theta_{3}=11.97^{\circ}$, then $r_{2 x}=-171.22 \mathrm{~mm}, \theta_{4 c}=\theta_{4 a}-\pi+\beta=11.97^{\circ}, \theta_{5}=-60^{\circ}$, and $r_{6}=250 \mathrm{~mm}$. Fig. 11(b) shows the corresponding dead-position configuration (line $\mathrm{L}_{2}$ ) of mode clamping mechanism at open position.

According to Figs. 11 (a) and 11 (b), the complete mechanism of mode clamping mechanism (I) with two dead-position configurations are shown in Figs. 12 (a) and 12 (b). 


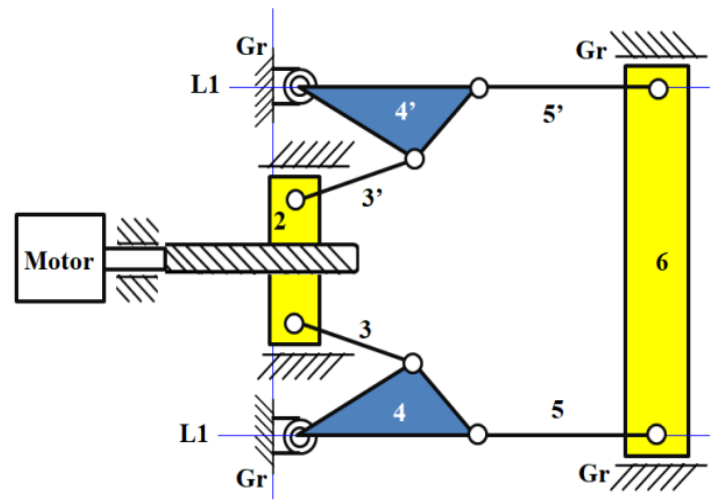

(a) Closed position (dead-position configuration, line L1)

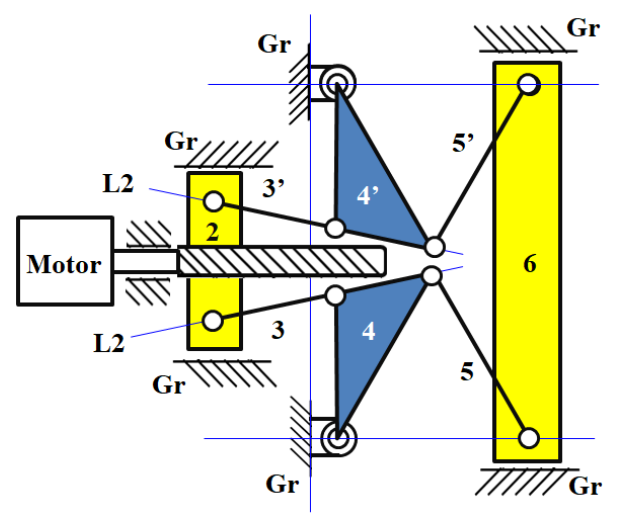

(b) Open position (dead-position configuration, line L2)

Fig. 12 Complete mechanism of mold clamping mechanism (I) with two dead-position configurations

\subsection{Case II}

Fig. 13 (a) and 13 (b) show the mode clamping mechanism and its corresponding vector coordinate system of the mechanism shown in Fig. 9 (b). According to Fig. 13 (b), the mechanism has two independent vector loops (vector loops 1 and 2). Vector loops 1 and 2 can be expressed as follows:

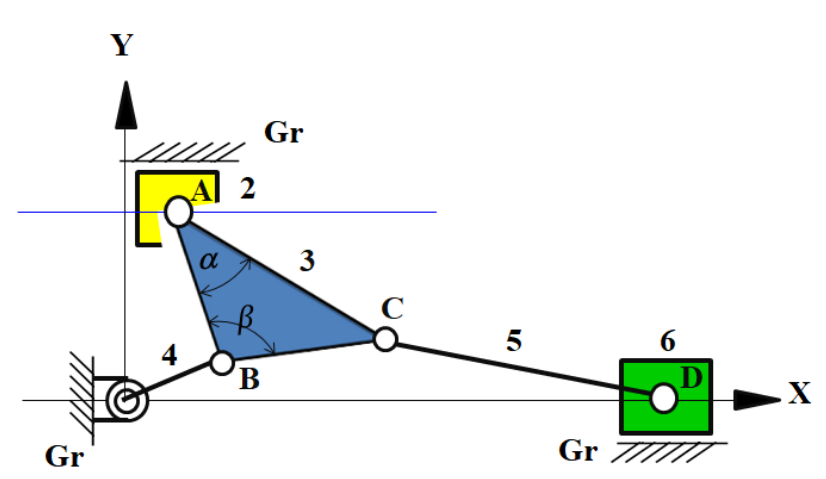

(a) Kinematic skeleton

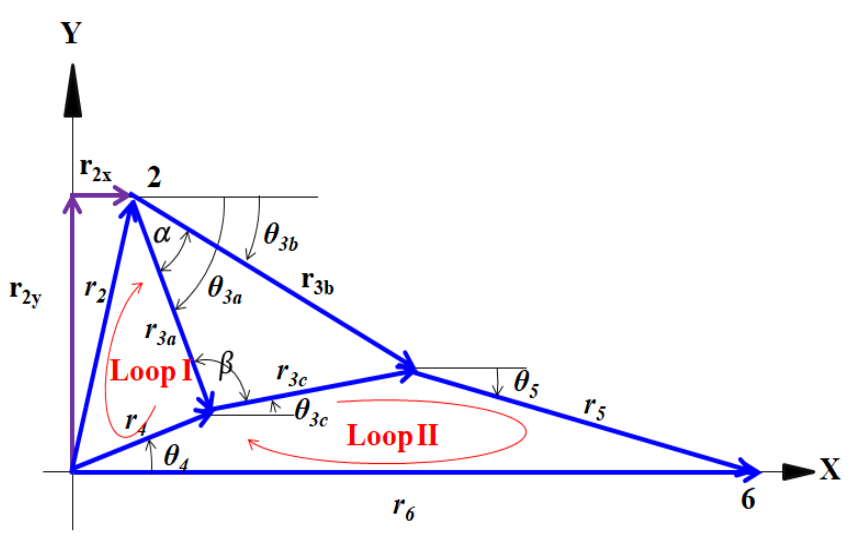

(b) Vctor coordinate system

Fig. 13 Mold clamping mechanism (II)

$$
\begin{aligned}
& \vec{r}_{2}+\vec{r}_{3 a}-\vec{r}_{4}=0 \\
& \vec{r}_{4}+\vec{r}_{3 c}+\vec{r}_{5}-\vec{r}_{6}=0
\end{aligned}
$$

According to Fig. 13 (b), $\theta_{3 c}=\theta_{3 a}+\pi-\beta$ are the given conditions. If we substitute them into the above equations, then the components $\mathrm{X}$ and $\mathrm{Y}$ can be expressed as:

$$
\begin{aligned}
& r_{2 x}+r_{3 a} \cos \theta_{3 a}-r_{4} \cos \theta_{4}=0 \\
& r_{2 y}+r_{3 a} \sin \theta_{3 a}-r_{4} \sin \theta_{4}=0 \\
& r_{4} \cos \theta_{4}+r_{3 c} \cos \theta_{3 c}+r_{5} \cos \theta_{5}-r_{6}=0
\end{aligned}
$$




$$
r_{4} \sin \theta_{4}+r_{3 c} \sin \theta_{3 c}+r_{5} \sin \theta_{5}=0
$$

If $r_{2 y}=162.8 \mathrm{~mm}, r_{4}=94 \mathrm{~mm}, r_{3 a}=162.8 \mathrm{~mm}, r_{3 c}=156 \mathrm{~mm}, r_{5}=250 \mathrm{~mm}$, and $\beta=90^{\circ}$, according to Eqs. (11) (14), we get:

(1) If $\theta_{4}=0^{\circ}$, then $\theta_{3 a}=-90^{\circ}, r_{2 x}=94 \mathrm{~mm}, \theta_{3 c}=\theta_{3 a}+\pi-\beta=0^{\circ}, \theta_{5}=0^{\circ}$, and $r_{6}=500 \mathrm{~mm}$. Fig. 14 (a) shows the corresponding dead-position configuration of mode clamping mechanism at closed position.

(2) If $\theta_{4}=60^{\circ}$, then $\theta_{3 a}=-30^{\circ}, r_{2 x}=-93.99 \mathrm{~mm}, \theta_{3 c}=\theta_{3 a}+\pi-\beta=60^{\circ}, \theta_{5}=-60^{\circ}$, and $r_{6}=250 \mathrm{~mm}$. Fig. 14 (b) shows the corresponding dead-position configuration of mode clamping mechanism at open position.

According to Figs. 14 (a) and 14 (b), the complete mechanism of mode clamping mechanism (II) with two dead-position configurations are shown in Figs. 15 (a) and 15 (b).

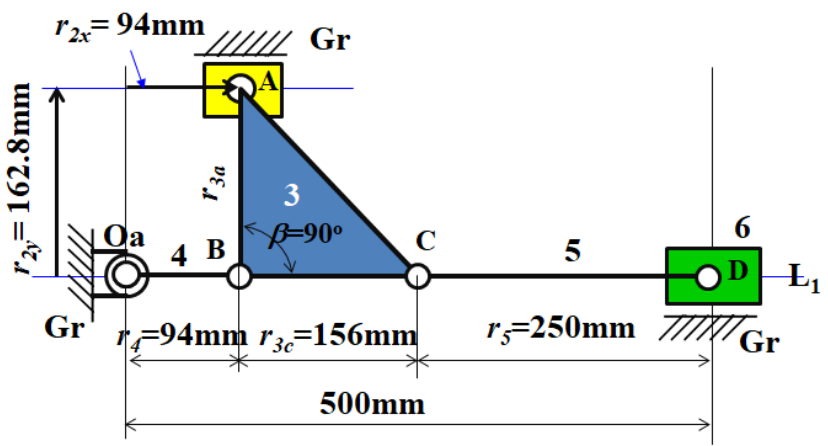

(a) Close position (dead-position configuration, line L1)

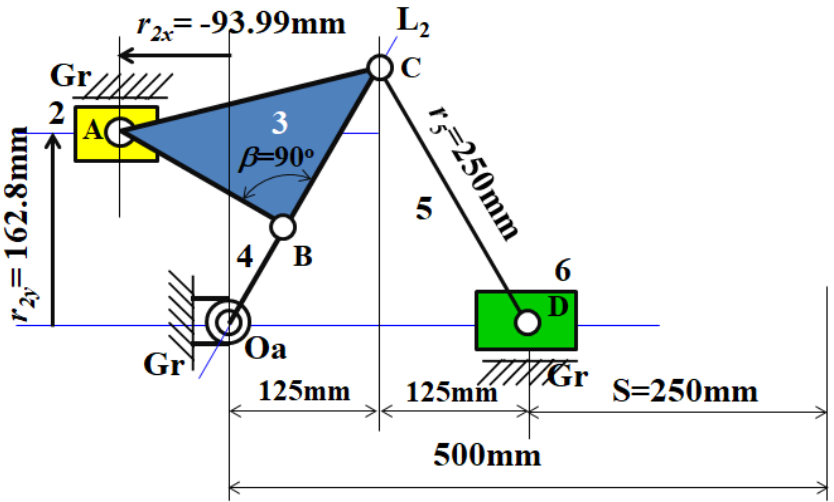

(b) Open position (dead-position configuration, line L2)

Fig. 14 Mold clamping mechanism (II) with two dead-position configurations

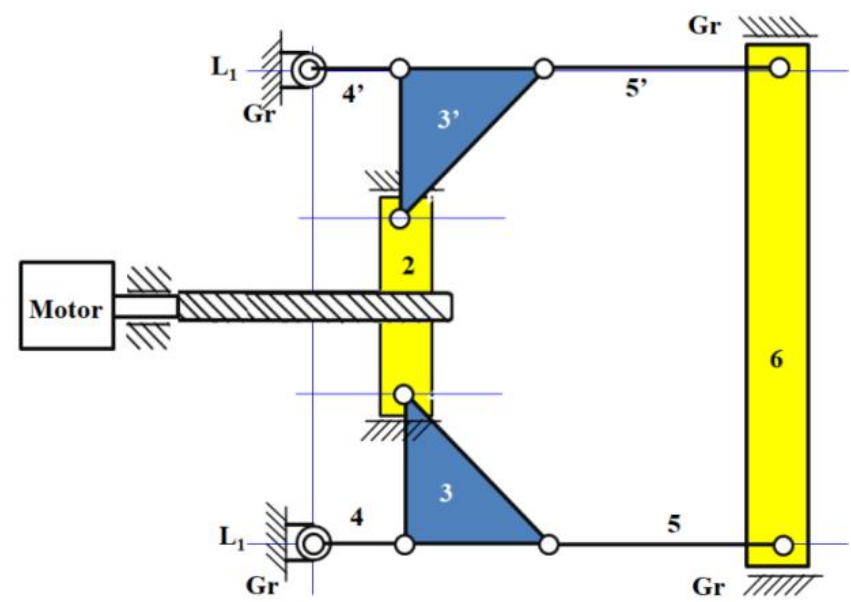

(a) Closed position (dead-position configuration, line L1)

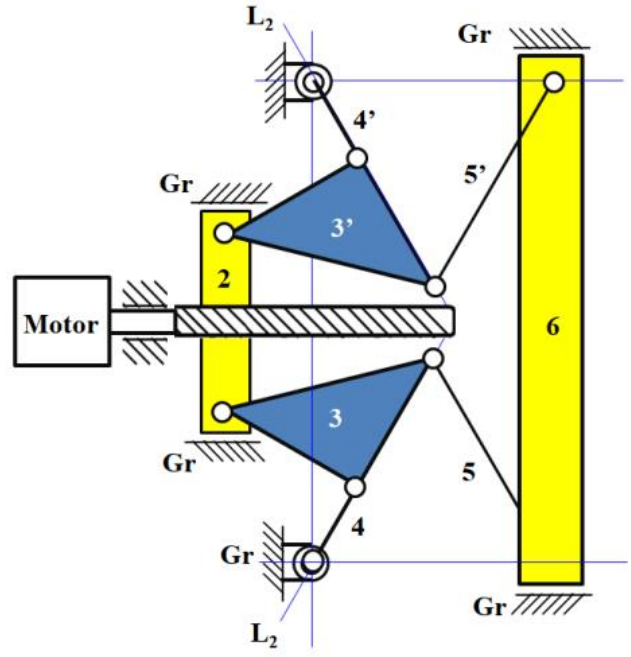

(b) Open position (dead-position configuration, line L2)

Fig. 15 Complete mechanism of mold clamping mechanism (II) with two dead-position configurations

\subsection{Case III}

Fig. 16 (a) and 16 (b) show the mode clamping mechanism and its corresponding vector coordinate system of the mechanism shown in Fig. 9 (c). According to Fig. 16 (b), the mechanism has two independent vector loops (vector loops 1 and 2). Vector loops 1 and 2 can be expressed as follows: 


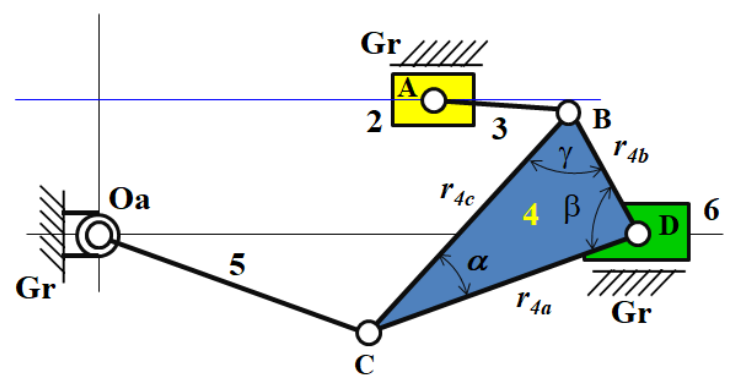

(a) Kinematic skeleton

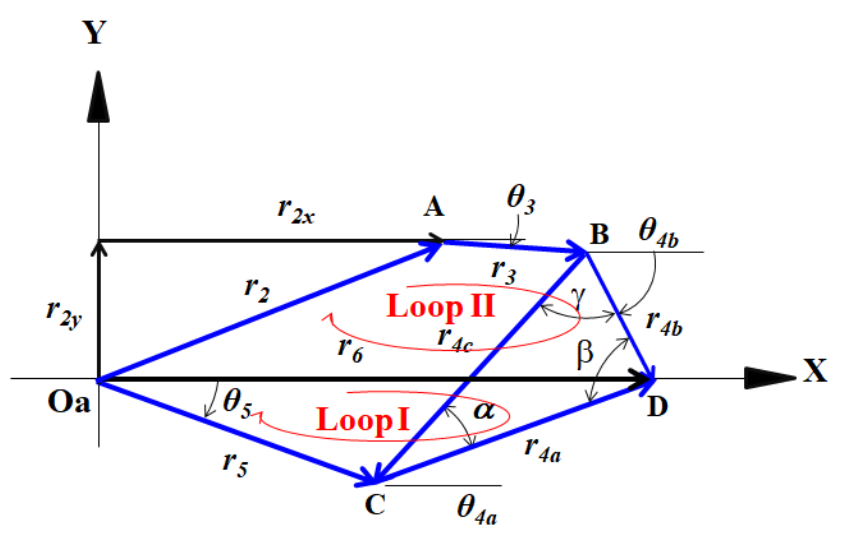

(b) Vector coordinate system

Fig. 16 Mold clamping mechanism (III)

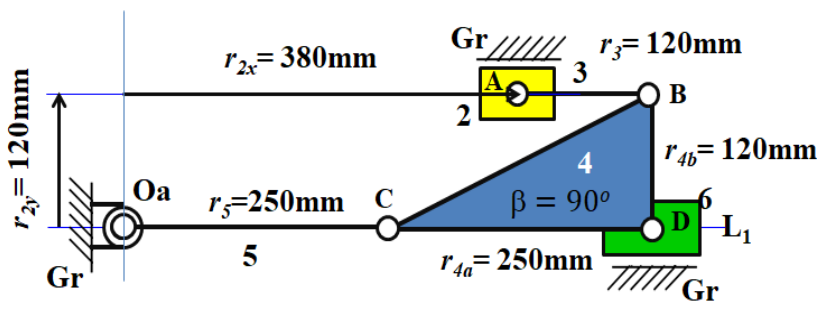

(a) Close position (dead-position configuration, line L1)

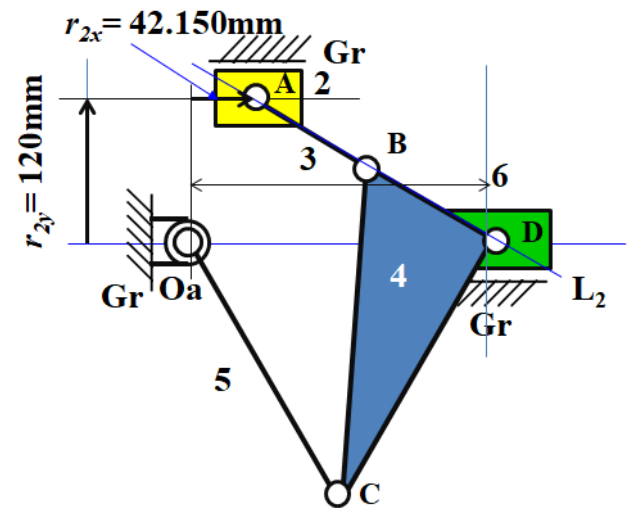

(b) Open position (dead-position configuration, line L2)

Fig. 17 Mold clamping mechanism (III) with two dead-position configurations

$$
\begin{aligned}
& \vec{r}_{5}+\vec{r}_{4 a}-\vec{r}_{6}=0 \\
& \vec{r}_{2}+\vec{r}_{3}+\vec{r}_{4 b}-\vec{r}_{6}=0
\end{aligned}
$$

According to Fig. 16(b), $\theta_{4 b}=\theta_{4 a}-\pi+\beta$ are the given conditions. If we substitute them into the above equations, then the components $\mathrm{X}$ and $\mathrm{Y}$ can be expressed as:

$$
\begin{aligned}
& r_{5} \cos \theta_{5}+r_{4 a} \cos \theta_{4 a}-r_{6}=0 \\
& r_{5} \sin \theta_{5}+r_{4 a} \sin \theta_{4 a}=0 \\
& r_{2 x}+r_{3} \cos \theta_{3}+r_{4 b} \cos \theta_{4 b}-r_{6}=0 \\
& r_{2 y}+r_{3} \sin \theta_{3}+r_{4 b} \sin \theta_{4 b}=0
\end{aligned}
$$

If $r_{2 y}=120 \mathrm{~mm}, r_{3}=120 \mathrm{~mm}, r_{4 a}=250 \mathrm{~mm}, r_{4 b}=120 \mathrm{~mm}, r_{5}=250 \mathrm{~mm}$, and $\beta=90^{\circ}$, according to Eqs. (17) (20), we get:

(1) If $\theta_{5}=0^{\circ}$, then $\theta_{4 a}=0^{\circ}, r_{2 x}=380 \mathrm{~mm}, \theta_{4 b}=\theta_{4 a}-\pi+\beta=-90^{\circ}, \theta_{3}=0^{\circ}$, and $r_{6}=500 \mathrm{~mm}$. Fig. 17(a) shows the corresponding dead-position configuration of mode clamping mechanism at closed position. 
(2) If $\theta_{5}=-60^{\circ}$, then $\theta_{4 a}=60^{\circ}, r_{2 x}=42.15 \mathrm{~mm}, \theta_{4 b}=\theta_{4 a}-\pi+\beta=-30^{\circ}, \theta_{3}=-30^{\circ}$, and $r_{6}=250 \mathrm{~mm}$. Fig. 17(b) shows the corresponding dead-position configuration of mode clamping mechanism at open position.

According to Figs. 17(a) and 17(b), the complete mechanism of mode clamping mechanism (III) with two dead-position configurations are shown in Figs. 18(a) and 18(b). According to Fig. 18(b), the mold is damaged because of too large stroke of the input slider, so it is not a good design.

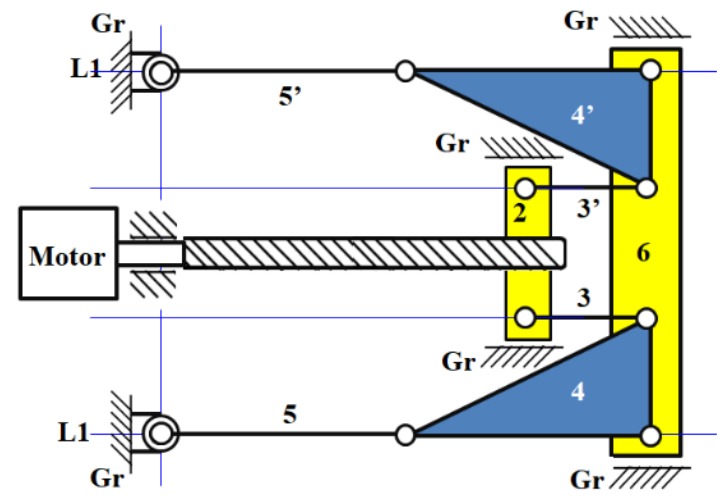

(a) Closed position (dead-position, line L1)

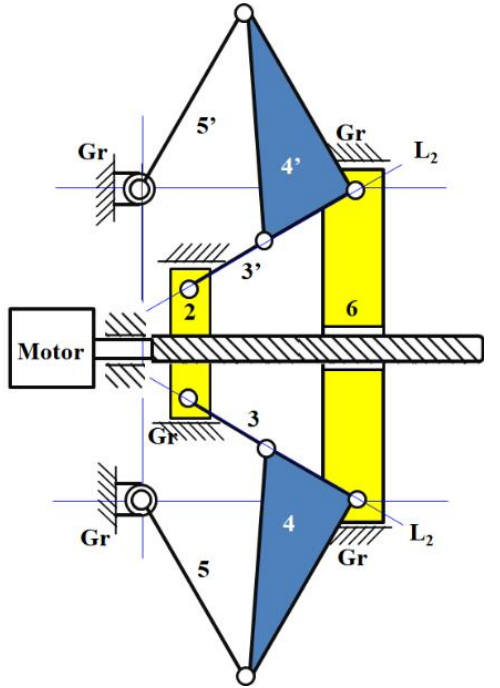

(b) Open position (dead-position, line L2)

Fig. 18 Complete mechanism of mold clamping mechanism (III) with two dead-position configurations

\section{Conclusions}

Traditionally, the mold clamping mechanism does not have dead-position configuration for the mold in the open position. This study focuses on the design of mold clamping mechanisms with two dead-position configurations when the mold is in open and closed positions. Firstly, by referring to the existing patents and the studies on the creative design, four feasible design concepts of mold clamping mechanisms are synthesized. Then, according to the studies on kinematics of mechanisms, three mold clamping mechanisms with two two dead-position configurations are also synthesized. Because of too large stroke of input slider, the design case III is not good. Only two mold clamping mechanisms with two two dead-position configurations (case I and case II) can be used to design the real mold clamping mechanism for linear blow-molding machine. The results of this research will enhance $R \& D$ capability of the mold clamping mechanisms to improve industrial competitiveness.

\section{Acknowledgement}

The authors are grateful to Chum-power Machinery Co., Ltd. for the support of this research under 107AF094.

\section{Conflicts of Interest}

The authors declare no conflict of interest.

\section{References}

[1] G. X. Lai, W. F. Su and G. X. Lin, The mode clamping mechanism of servo-drive type injection molding machine, Taiwan patent, 542097, Jul. 11, 2003.

[2] F. Q. Jiang, Driving device of controlling mode opening and closing for plastic molding machine, Taiwan patent, M257273, Feb. 21, 2005.

[3] W. T. Chang and L. Y. Wu, Ten-link clamping mechanism with double dead-positions, Taiwan patent, I382913, Jan. 21, 2013. 
[4] J. Li and G.W. Li, All-electric plastic injection machine, Taiwan patent, M510231, Oct. 11, 2015.

[5] W. Y. Lin, Y. C. Chang, Z. Cheng, X. R. Chen, and H. Y. Chen, New fast five-point locking mechanism with two dead-position configurations, Taiwan patent, M510233, Oct. 11, 2015.

[6] Y. Y Chen and J. C. Qin, Clamping device for mold, Taiwan patent, I537121, Jun. 11, 2016.

[7] W. Y. Lin, Y. C. Chang, and Y. J. Xiao, New five-point clamping mechanism with two dead-position configurations for injection molding machine, Taiwan patent, M541943, Mar. 21, 2017.

[8] H. S. Yan, “A methodology for creative mechanism design,” Mechanism and Machine Theory, vol. 27, no. 3, pp. 235-242, May. 1992.

[9] H. S. Yan, Creative Design of Mechanical Devices, Springer-Verlag, Singapore, 1998.

[10] W. H. Hsieh and S. J. Chen, "Innovative Design of Cam-Controlled Planetary Gear Trains," International Journal of Engineering and Technology Innovation, vol. 1, no. 1, pp. 01-11, 2011.

[11] L. C. Hsieh and T. H. Chen, "The Systematic Design of Link-Type Optical Fiber Polisher with Single Flat," Journal of Advanced Science Letters, vol. 9, pp. 318-324, 2012.

[12] G. S. Hwang and C. C. Lin, "Innovative Design of a Transmission Mechanism for Variable Speed Wind Turbines," JSME Journal of Advanced Mechanical Design, Systems, and Manufacturing, vol.9, no.3, pp. 1-11, 2015.

[13] T. H. Chen and L.C. Hsieh, "The Innovative Design of the Massage Mechanisms for Massage Chair," Advances in Technology Innovation, vol. 4, no. 2, pp. 116-124, 2019.

[14] Z. F. Yuan, M. J. Gilmartin, and S. S. Douglas, "Optimal Mechanism Design for Path Generation and Motions with Reduced Harmonic Content,” ASME Transactions, Journal of Mechanical Design, vol. 126, no. 1, pp. 191-196, 2004.

[15] W. H. Hsieh, "Kinematic synthesis of cam-controlled planetary gear trains," Mechanism and Machine Theory, vol. 44, no. 5, pp. 873-895, 2009.

[16] H. Li and Y. Zhang, "Seven-bar mechanical press with hybrid-driven mechanism for deep drawing; Part 1: Kinematic analysis and optimum design,” Journal of Mechanical Science and Technology, vol. 24, no.11, pp.2513-2160, 2010.

[17] W. H. Hsieh and C. H. Tsai, "On a Novel Press System with Six Links for Precision Deep Drawing," Mechanism and Machine Theory, vol. 46, no. 2, pp. 239-252, 2011.

[18] L. C. Hsieh and T. H. Chen, "The Systematic Design of Link-Type Optical Fiber Polisher with Single Flat," Journal of Advanced Science Letters, vol. 9, pp. 318-324, 2012.

[19] L. C. Hsieh and T. H. Chen, "The Systematic Design of Planetary-Type Grinding devices for Optical fiber ferrules and wafers," Transactions of the Canadian Society for Mechanical Engineering, vol. 40, no. 4, pp. 619-630, 2016.

[20] F. C. Chen, Y. F. Tzeng, and W. R. Chen, "On the Mechanism Design of an Innovative Elliptical Exerciser with Quick-Return Effect,” International Journal of Engineering and Technology Innovation, vol. 8, no. 3, pp. 228-239, 2018.

Copyright $\odot$ by the authors. Licensee TAETI, Taiwan. This article is an open access article distributed under the terms and conditions of the Creative Commons Attribution (CC BY-NC) license (https://creativecommons.org/licenses/by-nc/4.0/). 